Arbieva, Zarema

[11]

\section{Identification of dow nstream targets of the putative tumor suppressor gene on $8 p$ by differential gene expression analysis}

Zarema Arbieva, Kumarika Banerjee, Lydia Usha, Tiffany Le, Ignatius Gomes \& Carol Westbrook

University of Illinois, Chicago, Illinois, USA
Deletion mapping and functional studies indicate that chromosome 8 may contain a tumor suppressor gene involved in epithelial malignancies. Transfer of a human chromosome 8 results in suppression of the malignant phenotype in colon, breast and prostate cancer cell lines, which is attributed to reintroduction of the tumor suppressor gene. To study the mechanisms leading to these effects, we performed comparative expression analysis using GeneFilters arrays (Research Genetics). RNA from the HT-29 human colon cancer cell line was compared with its derivative containing chromosome 8, HT-29.X8, which demonstrates complete suppression of softagar clonicity and tumorigenicity compared with the parental line. The overall patterns of gene expression between the two cell lines changed only minimally; however, expression of four genes (RNF11, CED-6, KIAA0663 and PMEPA1) was increased after chromosome 8 introduction. Semiquantitative analysis by means of the polymerase chain reaction with reverse transcription confirmed that RNF11 and KIAA066 demonstrated a 1.5- to 2-fold increase; CED-6, a 2.5-fold increase; and $P M E P A 1$, an increase of over 20-fold. To determine the significance of these genes in breast cancer, their expression was analyzed in two breast cancer cell lines (MDAMB231 and ZR-75) and their derivatives containing chromosome 8. PMEPA1 showed 4- to 5-fold increase in the ZR-75 cells containing chromosome 8 , and KIAA0663 showed a 2.6-fold increase in the MDA-MB231 cells containing chromosome 8 . All these genes are probable downstream targets of the proposed $8 \mathrm{p}$ tumor suppressor gene, although some pathways may differ depending on the cell line. The biological relevance of these genes to carcinogenesis is the focus of ongoing studies.

Baas, Frank

\section{SAGE analysis of pediatric tumors identifies new markers and common genetic pathways}

Frank Baas, Esmee Oussoren, Anneloor ten Asbroek, Marjon Groenigen, Jennifer Benit-Deekman \& Marcel Kool

Department of Neurology, AMC Amsterdam, Amsterdam, The Netherlands

To identify genes that might be involved in the development and growth of medulloblastoma, rhabdomyosarcoma or both, we made Serial Analysis of Gene Expression (SAGE) libraries and compared the expression profiles of the tumors with those of normal tissue. In medulloblastoma we found OTX2 highly expressed and specific for this type of tumor. SAGE data were confirmed by northern blot analysis, and the majority of the medulloblastomas tested were positive for either OTX1 or OTX2. The OTX genes are orthologues of the Drosophila otd gene and are highly conserved in mammals. Immunohistochemistry with OTX2 antibodies confirmed the RNA data and showed that OTX expression is a good marker for medulloblastomas. We also identified HES6 expression in medulloblastomas and rhabdomyosarcomas. HES genes are orthologues of hairy, a transcription factor in Drosophila. Analysis of the CGAP SAGE libraries showed that expression of HES6 is rare in other tumors and tissues. Northern blot analysis of ten additional medulloblastomas and nine rhabdomyosarcomas confirmed the high rate of expression of HES-6 in the majority of these tumors. Expression of HES6 might be another marker for medulloblastoma and rhabdomyosarcoma.
Badie, Behnam

[13]

\section{Genomic analysis of low-grade gliomas using oligonucleotide arrays}

\author{
Behnam Badie, Sakthivel Prabakaran \& Jill Schartner
}

Department of Neurological Surgery, University of Wisconsin, Madison, Wisconsin, USA

With their potential for malignant transformation, low-grade gliomas of the brain demonstrate unpredictable biological behavior. To understand further the molecular pathogenesis of these tumors, we studied the broad pattern of gene expression in two freshly obtained low-grade oligodendrogliomas. Normal brain tissue harvested during epilepsy surgery was used as the control. Comparative analysis using oligonucleotide arrays (GeneChip, Affymetrix) revealed the overexpression of genes encoding the following proteins in both glioma specimens: bone morphogenetic protein (10- and 20-fold), Rb-binding protein, c-fos (17- and 35-fold), inhibitor of metalloproteinase 4 (14- and 24-fold), E2A transcription factor (12and 15-fold) and $b c l-1$ (44-fold). Based on this limited experience, we conclude that DNA microarray analysis is a powerful tool for studying the pathogenesis of low-grade gliomas. We plan to use this technology to analyze 30 additional lowgrade gliomas that are presently available through our brain tumor bank.

Baggerly, Keith

[14]

\section{Modeling significance and reproducibility on high-density CDNA microarrays}

Keith Baggerly1,2,3, Kevin Coombes ${ }^{1,2,4}$, Kenneth Hess ${ }^{1}$, David Stivers ${ }^{1,2}$, Lynne Abruzzo ${ }^{6} \&$ Wei Zhang ${ }^{2,5,7}$

${ }^{1}$ Department of Biostatistics and ${ }^{2}$ Cancer Genomics Program, M.D. Anderson Cancer Center, Houston, Texas, USA

${ }^{3}$ Department of Statistics, Rice University, Houston, Texas, USA

${ }^{4}$ Departments of Biomathematics, ${ }^{5}$ Pathology, and ${ }^{6}$ Hematopathology, M.D.

Anderson Cancer Center, Houston, Texas, USA

${ }^{7}$ Cancer Genomics Core Laboratory, M.D. Anderson Cancer Center, Houston, Texas, USA

A key problem in analyzing data from microarray experiments involves deciding when two expression levels corresponding to the same gene or expressed sequence tag are significantly different. A first approximation is to declare significance whenever the fold-difference between two expression levels exceeds some predefined threshold, but this approach ignores the fact that the variability of the ratio is linked to the overall expression level. A second approximation is to use multiple replicate arrays to build up an estimate of the standard deviation of the ratio on a gene-bygene basis, but this requires many arrays. We propose a middle ground. Beginning from first principles governing the behavior of loose strands of complementary DNA on microarrays, we derive a simple parametric model describing the type of variability that should be expected from chance alone, thus providing a null distribution for testing significance. When the variance of the log ratio is plotted as a function of the log intensity, the shape of the function is given by an exponential decay plus a constant. We estimate the parameters of the model on an array-byarray basis, using within-array replication, and then use replication between arrays to reduce the variation associated with our results. We illustrate the model using both simulations and data from a preliminary study of bladder cancer. 